\title{
Nucleation of Metal Nanoparticles on Amorphous Substrate: Insights into Orientation Preference and Heterogeneous Catalysis
}

\author{
Dipanwita Chatterjee ${ }^{1}$, Akash R. ${ }^{2}$, Kamalnath K. ${ }^{1}$, Rafia Ahmad ${ }^{1}$, Abhishek Singh $^{1}$ and N. Ravishankar ${ }^{1}$ \\ 1. Materials Research Centre, Indian Institute of Science, Bangalore, India \\ 2. Department of Materials Engineering, Indian Institute of Science, Bangalore, India
}

Hybrid nanostructures have found widespread application in heterogeneous catalysis ${ }^{1}$ and sensing. Improving the efficiency of the material has led to the extensive studies on the effect of the size and shape of the metal nanoparticles ${ }^{2,3}$ over the years. However, the effect of orientation of the metal nanoparticle with respect to the substrate has been overlooked as a parameter to control properties. The orientation of metal nanoparticle with respect to the substrate has profound impact on the activity of catalyst particles in terms of the total accessible surface area and the geometry of active site. In this paper, we have aimed at experimentally showing that particles that are heterogeneously nucleated show an orientation preference and that the preferred orientation is tunable; this experimental finding has also been justified using analytical calculations of the Winterbottom shape on substrates. We have also shown the implication of the orientation preference on carbon monoxide oxidation reaction catalysis by supported metal catalysts by DFT simulation.

Microwave reduction and nucleation of single crystalline $(<8 \mathrm{~nm})$ gold $(\mathrm{Au})$ and silver $(\mathrm{Ag})$ nanoparticles was done on carbon polymer substrates functionalized with different molecules. It was experimentally found that each of the systems had an orientation preference and the preference varied from one system to another due to change in the interfacial energy. Au nucleated on oleylaminefunctionalized carbon substrate and butanedithiol-functionalized carbon substrate had a nucleation orientation preference around [111] direction but the distribution in the orientation preference was much narrower in Au-butanedithiol system. Ag nucleated on oleylamine system on the other hand had a nucleation orientation around [011]. The representative TEM images of the Au and Ag nuclei on differently functionalized carbon substrates are shown in Fig 1. The orientation of each nucleus has been experimentally obtained indexing electron diffraction pattern obtained from each nucleus by Precession Electron Diffraction (PED) technique (as illustrated in Figure 2a-c). The experimental results on the orientations obtained using PED are graphically represented in Fig 2f.

Our analytical calculation for 3D FCC Winterbottom shapes shows that for a defined interfacial energy between an amorphous substrate and the FCC metal particle there exists one Winterbottom shape (one particular orientation) which is of lowest total surface energy and has the minimum nucleation barrier. If the difference in the nucleation barrier is more than the thermal energy $(\mathrm{kT})$, the preferred orientation can be realized experimentally. The tunability of the orientations is achieved by modifying the interfacial energy by functionalization.

Using DFT simulations we have shown the effect of orientation on geometric and steric factor of the catalyst particle taking the case of $\mathrm{CO}$ molecule adsorption on an active site of the catalyst. With change in the orientation of a nucleus for a given particle-substrate interface not only the total accessible area of the catalyst changes but also the position of the active site changes resulting in the dependence of the adsorption energy on the orientation of the catalyst particle. This result gives a direct implication of the importance of the study of the orientation preference in a heterogeneous nucleation of a metal 
nanoparticle [4].

\section{References:}

[1] Peterson, E. J. et al, Nature Commun. 5 (2014).

[2] Roldan Cuenya, B. Acc. Chem. Res. 46 (2012), p. 1682.

[3] Zhu, B. et al, Nano Lett. 16 (2016), p. 2628.

[4] Acknowledgement: The authors acknowledge DST for funding and AFMM facility of IISc.
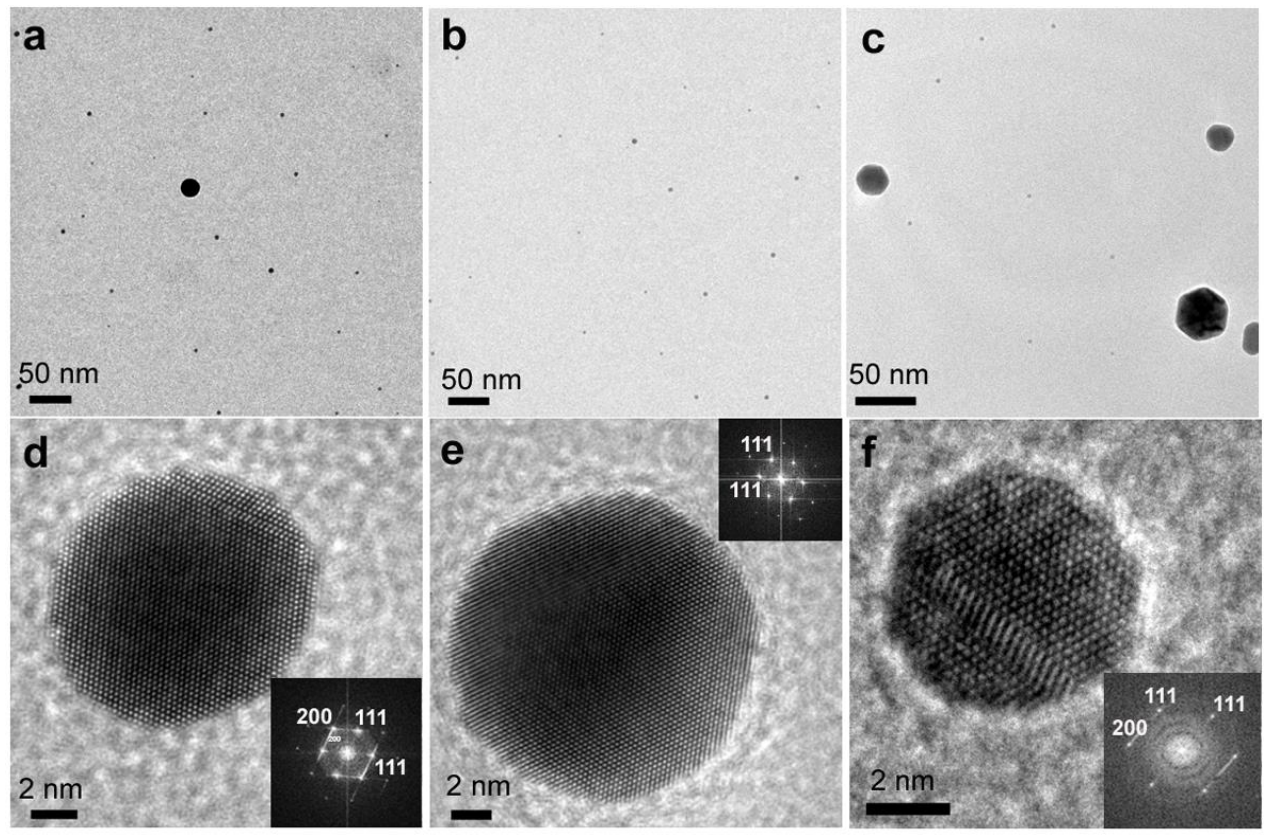

Figure 1. Low magnification images of Au-oleyl amine, Au-dithiol and Ag-oleylamine samples in a, b and $\mathrm{c}$ respectively and corresponding high resolution images of the respective samples showing single crystalline nuclei with twins/stacking faults in $\mathrm{d}$, e and $\mathrm{f}$.

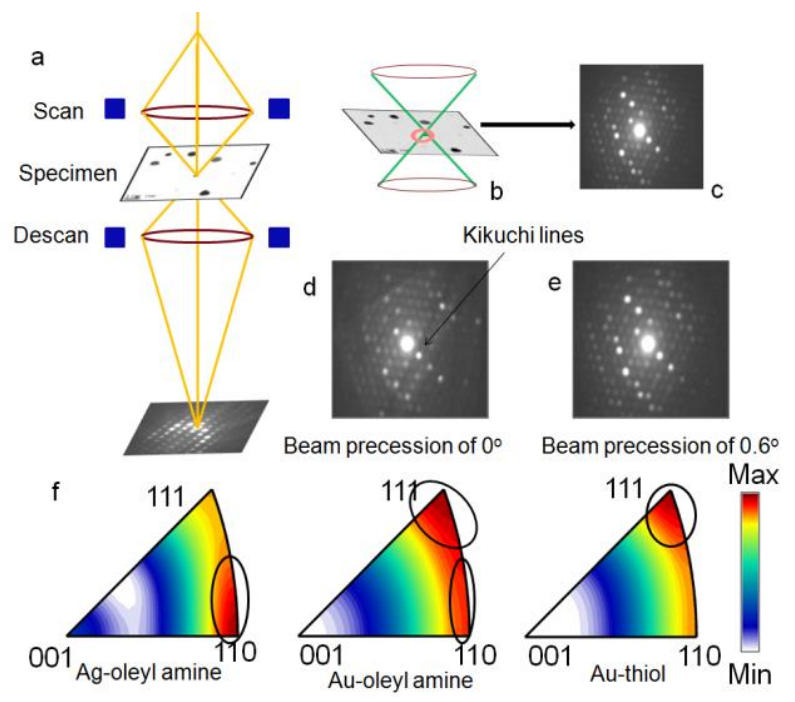

Figure 2. Schematic showing the working principle of Precession electron diffraction technique in a. A precessed beam used to acquire diffraction pattern $\mathrm{c}$ in the sample $\mathrm{b}$. Diffraction patterns in $\mathrm{d}$ and e acquired without and with precession respectively to show that intensity of the spots in the diffraction pattern in e is uniform, number of spots is more and Kikuchi lines disappear. Stereographic triangles in $\mathrm{f}$ showing maximum number of particles are having orientation around [110] direction in Ag-oleylamine, both Au-oleylamine and Au-thiol have maximum number of particles having orientation around [111] but the distribution is wider in Auoleylamine than in Au-thiol. 